\title{
A Review on the Study of Spinal Care and Spinal Motion
}

\author{
Yan Xiao, Wang Yanqun, Tian Hongmei \\ LinYi Normal University, LinYi, Shandong 276000, China
}

\begin{abstract}
This article reviews the health effects of spinal care and related movement of the spine. The spine is one of the important and the most sophisticated structure in the human body. Having healthy spine is having a healthy body. The normal spinal health mainly includes two aspects: One is enhancing the muscle strength of spine around, another is keeping the spine motion unit activities. The research's main purposes are to understand spine health and medicine, spine and spinal health, related sports as well as fitness exercises significance Review, which is in order to provide the theoretical basis for the spine health.
\end{abstract}

KEYWORD: Spine; health care; spinal motion

No matter in the field of Sports Sciences or in the medical field, spine studies are of great significance. The research take the spine health as the goal, combining the medical field of "spine" scientific theories and the research methods and the field of sports spinal motion method, to provide a theoretical basis for the code to create a scientific and effective spine exercises.

\section{THE RELATED MEDICAL RESEARCH OF SPINAL CARE}

After the Homo erectus, spine changes from the "beam" to "post", and it's continue to support the human body. In the process of conversion range, it is very hard to avoid to some uncomfortable symptom when the burden of spine increase, which there will be some spine problems if you don't pay attention to the spine care in the daily life. As the spine of the habitual posture is not correct, it will make the vertebral bearing unevenness, thereby increasing the burden on the spine, in the course of time, the intervertebral disc by extrusion and wear large, resulting in fibrous ring rupture. Disc is no longer closed, but rather to highlight the nucleus from the rupture, which is no stranger to the "lumbar disc herniation."The intervertebral disc is no longer sealed up, but the nucleus pulpous from rupture outstanding, which is not strange to everyone "lumbar disc herniation".

In addition, the present study found that more than 70 kinds of diseases are spine imbalance related to spine imbalance. These diseases involve human nervous, respiratory, digestive, urinary and endocrine and other systems, and many with spinerelated cases. Such as leg pain, mental changes, abnormal blood pressure, cholecystitis, bronchitis, coronary heart disease, hearing loss, dizziness, abdominal pain, chronic diarrhea[1]. These examples illustrate the importance of the spine in the human body.

\section{SIGNIFICANCE OF SPINE CARE}

The spine is one of the important structure of precision is most human, so having healthy spine is having a healthy body[2]. The first line of defense to ensure spinal health, is the right to treat the pain problem, good function of keeping the spine. The first line of defense to ensure spinal health, is the right to treat the pain problem, good function of keeping the spine. All soft body tissues and organs can "hang" get up, thanks to help skeletal system; and all the soft body tissues and organs can "hang" get up, thanks to the help of the skeletal system; and skeletal system bracket, all in the spine. On the top of the spine is the skull, hanging below the sacrum, the middle is also protecting the spinal cord, and peripheral nerves of the spinal cord is the main shaft, a pair of peripheral nerve from the middle of the spine body points out, then it moves to the left and right and up and down the distribution. Thirty-one pairs issued by the spinal nerve root dominate the internal organs and limbs movement. All the 
activities of the human body depend on the nervous system that has a close relationship with the spinal. Displacement of the vertebral body as well as vertebra oppression and even spinal muscle, ligament tissues or surrounding nervous, which will directly influence through nerve, indirect cause nerve supply at the end of the organs, muscle or secretory glands, leading to the organ dysfunction at last. Therefore, the spine is called "the body's second lifeline" by the medical experts[3].

\section{RESEARCH OF SPINAL MOVEMENT FITNESS EFFECT}

When the body being upright, the spine is "column; when it being lying down, the spine is the "beam, which are the name of this so-called origins of the spine and backbone. Spinal movement fitness results can be understood from the two angles: one is a normal spine care, enhance the muscle strength of spine around, keeping the spine motion unit activities, namely the spine flexibility, and the other is that there has been a neck and shoulder back pain symptoms of spine exercise, it can promote the rehabilitation, medical treatment can be used as a means of effective and beneficial supplement, especially in convalescence rehabilitation measures. In the research of spine-related diseases, cervical disease and lower back pain, many of which beneficial influence and promotion of the spinel movement[4].

Dai Kuiwu (2005), research suggests, neck muscles make the cervical stable, in orderto make it balance, ligament, joint and intervertebral disc connection. Degeneration andinner balance disorders bring up symptoms. The main role of self-movement therapy for this disease is through the neck muscles, strengthen the neck muscles in order to maintain the stability of the cervical spine; through the neck functional exercises, restore and enhance the activities of the cervical spine function to prevent cervical joint stiffness; neck and improve blood circulation, promote inflammation subsided, the neck muscles can also relieve muscle spasms to relieve pain, prevent muscle atrophy. At the same time self-exercise can struggle with the disease in patients with subjective initiative into full play. It can be seen from the results, self-exercise group significantly lower relapse rate than the control group, suggesting that long-term adherence selfexercise can significantly reduce the relapse rate, toconsolidate rehabilitation treatment[5].

\section{CONCLUSION}

Spine is the backbone of life. Spine, as the body's axial skeleton, its structure and movement of importance is self-evident. In today's society, the improvement of productivity in the human liberation from the heavy physical activity at the same time, however, it also deprived of the power of the human part of the manual labor. Modern lifestyle diseases, including cervical spondylosis, neck shoulder pain, low back pain and other spinal joint disease, which have become a frequent disease even with the onset of younger trend. Therefore, it is necessary to carry out the back of the spine scientific exercise. It has become a consensus that scientific and rational sport can effectively improve spinal joint disease and spine related diseases.

\section{ACKNOWLEDGEMENTS}

This work was supported by the natural science foundation of Shandong Province. Item Number is ZR2010HL001.

\section{REFERENCES}

[1] Yan Fang, Ting bin. Exercise spine prevent all diseases. People's sports press, 1999:3.

[2] Han Zhu, Decided to healthy cervical, thoracic and lumbar spine in life to centenarians. Wen Hui press, 2010:7.

[3] Li Yikai. Non-surgical treatment status quo of cervical spondylosis. Journal of practical medical journal, 2005, 21 (4): 340-345.

[4] Nan Dengkun, MiaoHongShi, Rehabilitation medicine. Beijing: people's medical publishing house, 1993.276277

[5] Lee Rielly, PT, Richard Holcombe, PT; Exercise for low back pain: the effect of patient-specific exercise prescriptions using progressive resistance exercises / The Spine Journal 2005 (5). 